\title{
Evolução clínica e funcional dos pacientes com infecção após artrodese de coluna lombar
}

\author{
Clinical and functional outcome of patients with deep wound \\ infection after spinal lumbar fusion \\ Evolución clínica y funcional de los pacientes con infección post- \\ artrodesis de columna lumbar
}

\author{
Asdrubal Falavigna' \\ Orlando Righesso ${ }^{2}$ \\ Alisson Roberto Teles ${ }^{3}$ \\ Fabrício Diniz Kleber ${ }^{4}$
}

\section{RESUMO}

Objetivos: descrever a experiência dos autores no manejo da infecção após instrumentação da coluna lombar e suas consequências futuras. Métodos: estudo de coorte prospectivo com pacientes portadores de infecção profunda pós-operatória, realizado entre janeiro de 1997 e janeiro de 2009. Todos os pacientes foram submetidos à revisão cirúrgica, coleta de material para exame microbiológico, lavagem exaustiva da região, debridamento dos tecidos desvitalizados, colocação de sistema de lavagem contínua, sutura primária e antibioticoterapia. Manteve-se a instrumentação em todos os pacientes. Os pacientes foram avaliados durante o seguimento por imagens radiográficas, estado clínico e funcional. Utilizaram-se a escala numérica da dor e o índice de incapacidade Oswestry. Os resultados numéricos foram submetidos a análises pareadas pelo teste de Wilcoxon. Resultados: a incidência de infecção foi de 3,1\% (15/485). $\mathrm{O}$ agente etiológico mais prevalente

\section{ABSTRACT}

Objectives: to describe the authors' experience in managing deep wound infection after surgical procedure on the lumbar spine, as well as to evaluate the clinical and functional long-term course of these patients. Methods: prospective cohort study, comprising patients who presented deep infection of the surgical wound between, carried out in January 1997 till January 2009. All the patients were submitted to surgical revision, collection of material for a microbiological examination, exhaustive washing, debridement, implementation of a continuous washing system, primary suturing of the wound and treatment with antibiotics. The instrumentation was not removed from any patient. The patients were evaluated radiologically, clinically and functionally during the followup. Paired analyses were performed by means of Wilcoxon test. Results: the incidence of infection was $3.1 \%$ (15/485). The most prevalent etiological agent was Staphylococcus aureus. All surgical procedures obtained
\end{abstract}

\section{RESUMEN}

Objetivos: describir la experiencia de los autores en el manejo de la infección post-instrumentación de la columna lumbar, bien como analizar sus consecuencias futuras. Métodos: estudio de cohorte prospectivo con los pacientes que presentaron infección profunda de herida operatoria entre Enero de 1997 y Enero de 2009. Todos los pacientes fueron sometidos a la revisión quirúrgica, colecta de material para análisis microbiológico, lavado exhaustivo, desbridamiento de los tejidos desvitalizados, colocación de sistema de lavado continuo, sutura primaria de la herida y antibióticoterapia. No se retiró la instrumentación en ningún paciente. Los pacientes fueron evaluados radiológicamente, clinicamente y funcionalmente en el seguimiento. Se realizaron análisis pareados con la prueba de Wilcoxon. Resultados: la incidencia de infección fue del 3.1\% (15/485). El agente etiológico más prevaleciente fue el Staphylococcus aureus. Si logro consolidación en todos los pacientes. En un seguimiento promedio de 47.6

\footnotetext{
Universidade de Caxias do Sul, Caxias do Sul (RS), Brasil.

'Doutor, Professor de Neurologia-Neurocirurgia da Universidade de Caxias do Sul (UCS), Caxias do Sul (RS), Brasil. ${ }^{2}$ Mestre, Ortopedista, Bento Gonçalves (RS), Brasil.

${ }^{3}$ Médico Residente da Universidade de Caxias do Sul (UCS), Caxias do Sul (RS), Brasil.

${ }^{4}$ Médico Residente da Universidade de Caxias do Sul (UCS), Caxias do Sul (RS), Brasil. 
foi o Staphylococcus aureus. Todos os casos lograram consolidação. Em um seguimento médio de 47,6 meses, observaram-se mudanças nos escores da escala numérica da dor $(\mathrm{p}=0,001)$ e índice de incapacidade Oswestry $(p=0,017) . \mathrm{Na}$ avaliação final, 64,2\% dos pacientes (9/14) apresentaram incapacidade mínima e 35,8\% (5/14), incapacidade moderada. Conclusão: o tratamento agressivo das infecções pós-operatórias de artrodese de coluna lombar permite evitar a retirada da instrumentação e manter a estabilidade vertebral. A despeito da complicação, os pacientes apresentaram melhoras em relação à dor e capacidade funcional pré-operatórias.

DESCRITORES: Infecção da ferida operatória; Coluna vertebral/ cirurgia; Complicações pósoperatórias; Procedimentos ortopédicos/instrumentação

consolidation. In a mean follow-up of 47.6 months, changes were seen in the numerical scale pain scores ( $p=0.001$ ), Oswestry Disability Index $(p=0.017)$ and physical component of SF-36 $(p=0.036)$. In the final evaluation, $64.2 \%$ of the patients (9/14) presented minimal disability, and 35.8\% (5/14), moderate disability. Conclusion: Aggressive treatment of postoperative infections after spinal lumbar fusion avoids removal of the instrumentation and allows the maintenance of the vertebral stability. Despite the complications, the patients presented improvement with regard to pain, functional capacity and preoperative quality of life.

KEYWORDS: Surgical wound infection; Spine/ surgery; Postoperative complications; Orthopedic procedures/instrumentation meses, se observaron alteraciones en los scores de la escala numérica del dolor $(p=0.001)$, indice de incapacidad Oswestry ( $p=0.017)$ y componente fisico del SF-36 ( $p=0.036)$. En la evaluación final, un $64.2 \%$ de los pacientes (9/14) presentaron incapacidad mínima y un 35.8\% (5/14), incapacidad moderada. Conclusión: el tratamiento agresivo de las infecciones postoperatorias de artrodesis de columna lumbar permite evitar la retirada de la instrumentación y mantener la estabilidad vertebral. A pesar de la complicación, los pacientes presentaron mejorías con relación al dolor, a la capacidad funcional y a la calidad de vida preoperatorias.
DESCRIPTORES: Infección de herida operatoria; Columna vertebral/ cirugía; Complicaciones postoperatorias; Procedimientos ortopédicos/instrumentación

\section{INTRODUÇÃO}

A incidência de infecções profundas de ferida operatória em cirurgias da coluna lombar situa-se entre 0,7 e 11,6\% ${ }^{1-6}$. Essa complicação resulta em hospitalização prolongada e aumento dos custos, podendo comprometer o resultado da cirurgia caso não seja manejada adequadamente ${ }^{7,8}$. A prevenção por meio de técnicas de assepsia e de antimicrobianos profiláticos continua sendo a alternativa mais adequada ${ }^{7,8}$.

Não há consenso na literatura em relação ao tratamento de escolha nas infecções após instrumentação da coluna lombar, em especial quanto a antibioticoterapia, lavagem externa, retirada da instrumentação, remoção do enxerto ósseo, fechamento por segunda intenção ou limpeza diária ${ }^{4-6,9-19}$. Além disso, a evolução clínica e funcional dos pacientes com infecção após instrumentação tem sido pouco relatada na literatura ${ }^{20,21}$.

O objetivo deste estudo foi descrever a experiência dos autores em relação ao manejo da infecção após instrumentação da coluna lombar, bem como avaliar a evolução clínica e funcional tardia desses pacientes.

\section{METODOS}

\section{Delineamento e casuística}

Estudo de coorte prospectivo com pacientes que apresentaram infecção cirúrgica após artrodese de coluna lombar por via posterior durante o período de Janeiro de 1997 a Janeiro de 2009. Os pacientes foram avaliados no pré-operatório e no seguimento, com instrumentos que avaliavam nível de dor e capacidade funcional.

As cirurgias foram realizadas pela mesma equipe em todos os casos (AF, OR). Todos pacientes realizaram antibioticoprofilaxia com cefazolina durante o primeiro procedimento cirúrgico. O diagnóstico de infecção foi definido pelo crescimento bacteriano no líquido da ferida operatória.

\section{Manejo da infecção cirúrgica}

Os pacientes com suspeita clínica de infecção da ferida operatória foram submetidos à revisão cirúrgica, lavagem exaustiva com coleta de material para exame bacteriológico e remoção do tecido necrótico. Os fragmentos ósseos superficiais, que estavam desvitalizados, foram retirados; entretanto, os mais profundos foram deixados para obtenção de uma consolidação óssea. O material de fixação foi mantido, sendo instalado um sistema de lavagem contínua com um dreno de Portovac ligado ao equipo de solução fisiológica a $0,9 \%$, com gotejo de 40 gotas por minuto, e um dreno de tórax conectado ao sistema de frascos coletores, ligados à rede de vácuo para retirada do líquido. Realizou-se a sutura primária da ferida operatória em todos os casos. O sistema de lavagem foi mantido por cinco dias, podendo ser recolocado conforme a ne- 
cessidade. Ao mesmo tempo, foi instituído antibioticoterapia intravenosa seguida por via oral, de acordo com a sensibilidade do micro-organismo.

\section{Avaliações clínicas e funcionais}

A resposta ao tratamento e a duração da antibioticoterapia foram monitoradas por meio de leucograma, velocidade de sedimentação globular e proteína $\mathrm{C}$ reativa. Todos os pacientes foram submetidos a exame radiográfico funcional no seguimento para avaliar a artrodese.

O nível de dor foi avaliado pela escala numérica da dor (Numerical Rating Scale, NRS), que avalia a dor do paciente em uma escala de 0 (sem dor) a 10 (máxima dor possível). Considera-se como mudança mínima clínica importante uma variação de 2 pontos ou $30 \%$ da escala ${ }^{22}$.

A capacidade funcional foi avaliada pelo índice de incapacidade Oswestry (Oswestry Disability Index, ODI). O ODI é um questionário autoaplicável, que mede incapacidade em uma escala de dez itens, com seis categorias de resposta; cada item varia de 0 a 5 . Os escores são transformados em uma escala de 0 a 100. Pacientes com escore de 0 a 20 têm incapacidade mínima; de 21 a 40, incapacidade moderada; de 41 a 60, incapacidade grave; de 61 a 80, estão inválidos; e de 81 a 100, estão acamados ou exagerando seus sintomas ${ }^{23}$. Considera-se como mudança mínima clinicamente importante uma variação de 10 pontos ou $30 \%$ do escore tota ${ }^{22}$.

\section{Análise estatística}

As análises estatísticas foram conduzidas com o Statistical Package for the Social Sciences (SPSS) 16.0. As variáveis categóricas foram apresentadas como número e proporção. As variáveis contínuas foram apresentadas como média e desvio padrão. Para verificar mudanças entre os escores dos instrumentos no seguimento em relação ao pré-operatório foram conduzidas análises pareadas com o teste de Wilcoxon. Significância estatística foi considerada quando $\mathrm{p}<0,05$.

\section{RESULTADOS}

A infecção pós-operatória foi observada em $15(3,1 \%)$ dos 485 casos operados para fixação posterior lombar, com instrumentação e enxertia óssea de ilíaco nos últimos 12 anos. A idade média dos pacientes acometidos foi 59,8 anos (39-74), e 53\% eram do gênero masculino (8/15). A Tabela 1 demonstra as características dos pacientes inseridos no estudo.

A maioria dos pacientes apresentou o início dos sintomas entre uma e quatro semanas após a cirurgia. Apenas um caso apresentou sintomatologia tardia (36 semanas). Todos apresentaram secreção na ferida operatória com crescimento de micro-organismos no exame cultural. Foi necessário recolocar o sistema de lavagem em dez casos, sendo que dois deles necessitaram destedesse tratamento por três vezes. O agente etiológico mais prevalente foi o Staphylococcus aureus $(80 \% ; 12 / 15)$. Antibioticoterapia intravenosa foi mantida por quatro a seis semanas, seguida por via oral, no máximo durante duas semanas. O tempo médio de hospitalização dos pacientes foi de 38,7 dias (28 a 49 dias).

\section{Evolução}

O seguimento médio dos pacientes foi de 47,6 meses (variação 6-150; desvio padrão: 4,51). Dos 15 pacientes que apresentaram a infecção da ferida operatória, oito responderam o questionário ODI no pré-operatório e 14, no pósoperatório. Todos os pacientes responderam o NRS no pré e no seguimento. Os dados referentes ao nível de dor e incapacidade estão apresentados na Tabela 2.

Dos oito pacientes que responderam o ODI no préoperatório e no seguimento, $75 \%$ (6/8) apresentaram variação de pelo menos $30 \%$ no ODI. Todos pacientes apresentaram diferença mínima clinicamente significante do NRS. As análises pareadas demonstraram diferença estatisticamente significativa entre os escores do NRS $(p=0,001)$ e do ODI $(p=0,017)$ no seguimento em comparação com o pré-operatório. Dos 14 pacientes avaliados com o ODI no seguimento, $64,2 \%$ dos pacientes $(9 / 14)$ apresentaram incapacidade mínima e 35,8\% (5/14), incapacidade moderada.

Todos pacientes apresentaram consolidação óssea póstero-lateral, ou seja, a presença da infecção não prejudicou a formação do calo ósseo.

\section{DISCUSSÃO}

O manejo da infecção de ferida operatória tem sido motivo de intenso debate na literatura. As consequências dessa complicação podem ser devastadoras para o paciente. Nosso estudo verificou incidência de $3,1 \%$ de infecção em pacientes submetidos à artrodese via posterior com instrumentação e enxerto ilíaco. A despeito dessa complicação, observaram-se mudanças importantes no nível de dor e na incapacidade dos pacientes em um seguimento médio de 46,7 meses. Mok et al..$^{21}$, em estudo de coorte comparando 16 pacientes com infecção de ferida operatória com 16 controles, demonstraram resultados funcionais semelhantes para os dois grupos em um seguimento médio de 56,7 meses.

Múltiplos fatores de risco para infecção após artrodese lombar têm sido descritos na literatura. A desnutrição parece ser um dos fatores principais. A baixa ingestão proteica está associada à imunossupressão e à dificuldade de cicatrização, sendo ideal que o nível de albumina seja superior a $3,5 \mathrm{~g} / \mathrm{dL}$ e que a contagem de linfócitos se situe entre 1.500 e 2.000 células $/ \mathrm{mm}^{3}$ para que os riscos de infecção sejam reduzidos ${ }^{7,24}$. Os pacientes fumantes têm incidência nove vezes maior de apresentar infecção quando comparados aos não fumantes ${ }^{9}$. Outros fatores descritos são obesidade, diabetes, repouso prolongado, imunossupressão, patologia tumoral, cirurgia prévia e infecção em outro sítio $^{10,25}$. A idade isolada não parece ser um fator preditor de infecção ${ }^{19,26}$. Fatores relacionados à cirurgia também levam a um maior risco de infecção pós-operatória, sendo eles: duração superior a cinco horas, retração prolongada, 


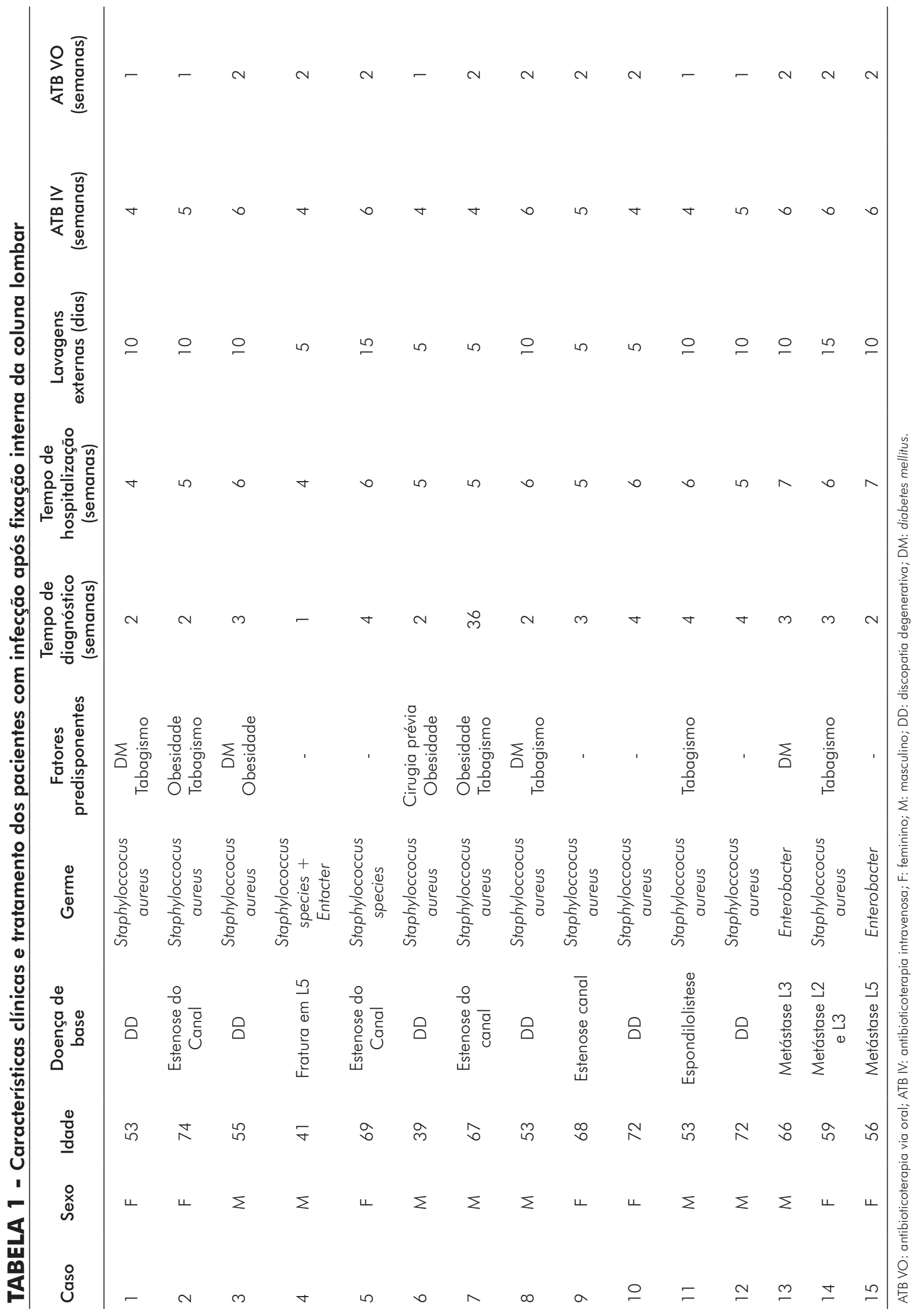


TABELA 2 - Evolução dos pacientes com infecção após artrodese lombar

\begin{tabular}{|c|c|c|c|c|c|}
\hline \multirow{2}{*}{ Pacientes } & \multirow{2}{*}{ Seguimento (em meses) } & \multicolumn{2}{|c|}{ Dor } & \multicolumn{2}{|c|}{ Oswestry } \\
\hline & & Pré & Final & Pré & Final \\
\hline 1 & 6 & 10 & 2 & 54 & 16 \\
\hline 2 & 12 & 9 & 0 & 38 & 2 \\
\hline 3 & 12 & 8 & 2 & 26 & 28 \\
\hline 4 & 12 & 8 & 2 & 38 & 26 \\
\hline 5 & 12 & 9 & 1 & 34 & 28 \\
\hline 6 & 30 & 9 & 4 & 40 & 24 \\
\hline 7 & 26 & 9 & 3 & 34 & 20 \\
\hline 8 & 29 & 10 & 2 & 58 & 16 \\
\hline 9 & 36 & 8 & 1 & - & 0 \\
\hline 10 & 36 & 9 & 3 & - & 0 \\
\hline 11 & 40 & 8 & 1 & - & 20 \\
\hline 12 & 108 & 10 & 2 & - & 24 \\
\hline 13 & 95 & 10 & 2 & - & 16 \\
\hline 14 & 110 & 9 & 6 & - & 14 \\
\hline 15 & 150 & 8 & 3 & - & - \\
\hline Total & $47,6( \pm 4,51)$ & $8,93( \pm 0,79)$ & $2,27( \pm 1,43)$ & $40,25( \pm 10,66)$ & $16,71( \pm 9,81)$ \\
\hline
\end{tabular}

tráfego excessivo de pessoas na sala cirúrgica, uso de enxertos ósseos, perda sanguínea acima de $1.000 \mathrm{~mL}$, tempo de hospitalização prévio à cirurgia e uso de instrumentação ${ }^{9,27-30}$. Fatores predisponentes foram observados em $60 \%$ dos pacientes nesse estudo.

O sucesso terapêutico depende do diagnóstico preco$\mathrm{ce}^{6,19}$. No pós-operatório, os pacientes queixam-se de desconforto lombar relacionado à dissecção muscular, podendo estar associado a temperatura elevada, edema, eritema ou descamação da ferida operatória ${ }^{1,2}$. A drenagem de secreção purulenta pela ferida operatória é tardia, estando presente em $93 \%$ dos pacientes no momento do diagnóstico ${ }^{1,19}$. Em nossa casuística, todos pacientes apresentaram drenagem de secreção purulenta pela ferida. Na maioria dos pacientes, os sinais de infecção se apresentaram entre uma e quatro semanas pós-operatórias, corroborando dados da literatura ${ }^{15}$.

Os exames laboratoriais, como leucograma, velocidade de sedimentação globular e proteína $\mathrm{C}$ reativa, são sensíveis - porém, não específicos, podendo ser parâmetros no estabelecimento do diagnóstico de infecção e na eficácia do tratamento ${ }^{19,28}$. Em nossa experiência, esses parâmetros laboratoriais são mais úteis no controle do tratamento dos pacientes com infecção $0^{6}$. Os estudos de imagem por tomografia computadorizada e ressonância magnética têm limitação pela presença de artefato dos dispositivos metálicos ${ }^{3,7}$.

Staphylococcus aureus foi o micro-organismo mais comumente associado à infecção de ferida operatória em nosso estudo, corroborando outros estudos da literatura ${ }^{3,7,8,10,31}$. Outros germes têm sido apontados como responsáveis pelas infecções, como o Staphylococcus epidermidis, Enterobacter cloacae, Escherichia coli e Enterococcus, sendo comuns as associações de Staphylococcus aureus e Peptococcus, Staphylococcus aureus e Enterococcus, Staphylococcus epidermidis e Pseudomonas aeruginosa ${ }^{2,29}$.
Todos os pacientes deste estudo apresentaram resolução da infecção após tratamento agressivo com reabertura da ferida, lavagem exaustiva, debridamento, sistema de irrigação contínua, fechamento primário e antibioticoterapia. Outros autores também relatam bons resultados clínicos com essa mesma abordagem ${ }^{2,3}$. O sistema de irrigação contínua deve ser mantido por cinco a dez dias, podendo ser repetido conforme a necessidade $e^{6-8}$. Todos os pacientes devem receber antibioticoterapia por cerca de seis semanas, de acordo com os testes de sensibilidade aos antimicrobianos, e um novo debridamento deve ser realizado se houver necessidade, dependendo da extensão e da aparência da lesão e do micro-organismo causador ${ }^{3,7,8,31}$. Outros autores adotam a irrigação com solução salina e bacitracina durante o procedimento inicial e descartam a necessidade do uso de sistemas de sucção e irrigação pósoperatórios ${ }^{1,18,32,33}$. Estes sistemas podem acarretar maior risco de pseudoartose e superinfecção por Pseudomonas, assim como impossibilitar a realização de debridamento de tecido necrótico se necessário ${ }^{16,34}$

A principal questão a ser esclarecida sobre a terapia da infecção pós-operatória é a remoção ou não da instrumentação. $\mathrm{O}$ uso da instrumentação de coluna lombar por via posterior elevou o índice de infecção de 2 para $6 \% \%^{3,10}$. Weinstein, McCabe e Cammisa ${ }^{1}$ afirmam que a instrumentação e os enxertos ósseos devem ser deixados para elevarem ao máximo a chance de fusão. Esta conduta não é consenso, já que, em $35 \%$ dos casos com infecção de coluna lombar, é necessária a remoção da instrumentação para aumentar a chance de erradicação da infecção, devido à proliferação de fibroblastos e reação de corpo estranho ${ }^{3,11,12}$. Outros autores preconizam a remoção não só da instrumentação como também do enxerto ósseo $0^{1,4,5,7,16,17}$. Nas infecções tardias, considera-se que a remoção da 
instrumentação é mais importante em razão da formação de um "glicocálice", que não pode ser removido sem a retirada da instrumentação, sendo que ele não apenas inibe o sistema imunológico, como também impede a detecção dos micro-organismos presentes na instrumentação com as técnicas de aspiração e culturas usadas. O tratamento efetivo nas infecções tardias inclui a irrigação, debridamento, remoção da instrumentação, uso de drenos e administração de antibióticos por seis semanas ${ }^{13-15}$. Por outro lado, a instrumentação serve para estabilizar o segmento comprometido, diminuindo, teoricamente, a inflamação e acelerando a cicatrização ${ }^{7}$. Existem muitos artigos na literatura discordando da conduta de remoção da instrumentação, por prejudicar muito o resultado da cirurgia ${ }^{4,5,16,17}$. A instrumentação não deve ser retirada em situações agudas, sendo mantida para atingir a imobilização desejada, uma vez que desalinhamento da coluna, compressão neural e paralisia são complicações devastadoras associadas à perda de estabilidade, quando o equipamento é removido antes do tempo para adequada fusão ${ }^{18}$. Quando a artrodese estiver consolidada, a instrumentação pode ser removida se necessário ${ }^{8}$. Pseudoartrose pode vir a ocorrer após uma infecção profunda em 30 a $85 \%$ dos pacientes ${ }^{4}$. Também pode ser apontada como causa de infecção, havendo associação ainda não bem estabelecida entre pseudoartrose e infecção cirúrgica ${ }^{35}$.

\section{CONCLUSÃO}

Esse estudo demonstrou que o manejo agressivo com abertura da ferida operatória, lavagem exaustiva, debridamento dos tecidos desvitalizados associado a um sistema de lavagem contínua, fechamento primário da ferida e antibioticoteapia é eficaz no tratamento da infecção e na manutenção da instrumentação. Além disso, em relação ao pré-operatório, foram observadas alterações significativas na dor, funcionalidade e qualidade de vida dos pacientes. A maioria dos pacientes apresentava incapacidade mínima no seguimento médio de 47,6 meses.

\section{REFERÊNCIAS}

1. Weinstein MA, McCabe JP, Cammisa FP Jr. Postoperative spinal wound infection: a review of 2,391 consecutive index procedures. J Spinal Disord. 2000;13(5):422-6.

2. Glassman SD, Dimar JR, Puno RM, Johnson JR. Salvage of instrumental lumbar fusions complicated by surgical wound infection. Spine. 1996;21(18):2163-9.

3. Abbey DM, Turner DM, Warson JS, Wirt TC, Scalley RD. Treatment of postoperative wound infections following spinal fusion with instrumentation. J Spinal Disord. 1995;8(4):278-83.

4. Stambough JL, Beringer D. Postoperative wound infections complicating adult spine surgery. J Spinal Disord. 1992;5(3):277-85.

5. Massie JB, Heller JG, Abitbol JJ, McPherson D, Garfin SR. Postoperative posterior spinal wound infections. Clin Orthop Relat Res. 1992;(284):99-108.

6. Falavigna A, Righesso Neto O, Fonseca GP, Nervo M. Manejo da infecção após cirurgia de fixação interna da coluna lombar. Arq Neuropsiquiatr. 2006;64(4):1001-4.

7. Beiner JM, Grauer J, Kwon BK, Vaccaro AR. Postoperative wound infections of the spine. Neurosurg Focus. 2003;15(3):E14. Review.
8. Picada R, Winter RB, Lonstein JE, Denis F, Pinto MR, Smith MD, et al. Postoperative deep wound infection in adults after posterior lumbosacral spine fusion with instrumentation: incidence and management. J Spinal Disord. 2000;13(1):42-5.

9. Thalgott JS, Cotler HB, Sasso RC, LaRocca H, Gardner V. Postoperative infections in spinal implants. Classification and analysis--a multicenter study. Spine. 1991;16(8):981-4.

10.Hodges SD, Humphreys SC, Eck JC, Covington LA, Kurzynske NG. Low postoperative infection rates with instrumented lumbar fusion. South Med J. 1998;91(12):1132-6.

11. Cordero J, Munuera L, Folgueira MD. Influence of metal implants on infection. An experimental study in rabbits. J Bone Joint Surg Br. 1994;76(5):717-20.

12.Galante JO, Lemons J, Spector M, Wilson PD Jr, Wright TM. The biologic effects of implant materials. J Orthop Res. 1991;9(5):760-75.

13. Gristina AG, Costerton JW. Bacterial adherence to biomaterials and tissue. The significance of its role in clinical sepsis. J Bone Joint Surg Am. 1985;67(2):264-73.

14.Richards BS. Delayed infections following posterior spinal instrumentation for the treatment of idiopathic scoliosis. J Bone Joint Surg Am. 1995;77(4):524-9.
15. Viola RW, King HA, Adler SM, Wilson CB. Delayed infection after elective spinal instrumentation and fusion. A retrospective analysis of eight cases. Spine. 1997;22(20):244450; discussion 50-1.

16.Dernbach PD, Gomez H, Hahn J. Primary closure of infected spinal wounds. Neurosurgery. 1990;26(4):707-9.

17.Griffiths HE, Jones DM. Pyogenic infection of the spine. A review of twenty-eight cases. J Bone Joint Surg Br. 1971;53(3):383-91.

18.Levi AD, Dickman CA, Sonntag VK. Management of postoperative infections after spinal instrumentation. J Neurosurg. 1997;86(6):975-80.

19.Lonstein J, Winter R, Moe J, Gaines D. Wound infection with Harrington instrumentation and spine fusion for scoliosis. Clin Orthop Relat Res. 1973;(96):222-33.

20.Mirovsky Y, Floman Y, Smorgick Y, Ashkenazi E, Anekstein Y, Millgram MA, et al. Management of deep wound infection after posterior lumbar interbody fusion with cages. J Spinal Disord Tech. 2007;20(2):127-31.

21.Mok JM, Guillaume TJ, Talu U, Berven SH, Deviren V, Kroeber M, et al. Clinical outcome of deep wound infection after instrumented posterior spinal fusion: a matched cohort analysis. Spine. 2009;34(6):578-83. 
22. Ostelo RW, Deyo RA, Stratford P, Waddell G, Croft P, Von Korff M, et al. Interpreting change scores for pain and functional status in low back pain: towards international consensus regarding minimal important change. Spine. 2008;33(1):90-4.

23. Fairbank JC, Pynsent PB. The Oswestry Disability Index. Spine. 2000;25(22):2940-52; discussion 2952. Comment in: Spine. 2000;25(21):2486-7.

24.Klein J, Garfin S. Nutritional status in the patient with spinal infection. Orthop Clin North Am. 1996;27(1):105-10.

25.Simpson JM, Silveri CP, Balderston RA, Simeone FA, An HS. The results of operations on the lumbar spine in patients who have diabetes mellitus. J Bone Joint Surg Am. 1993;75(12):1823-9.

26.Capen DA, Calderone RR, Green A. Perioperative risk factors for wound infections after lower back fusions. Orthop Clin North Am. 1996;27(1):83-6.
27.Pittet D, Ducel G. Infectious risk factors related to operating rooms. Infect Control Hosp Epidemiol. 1994;15(7):456-62.

28. Thelander U, Larsson S. Quantitation of C-reactive protein levels and erythrocyte sedimentation rate after spinal surgery. Spine. 1992;17(4):400-4.

29. Olsen MA, Mayfield J, Lauryssen C, Polish LB, Jones M, Vest J, et al. Risk factors for surgical site infection in spinal surgery. J Neurosurg. 2003;98(2 Suppl):149-55.

30.Wimmer C, Guch H, Franzreb M, Ogon M. Predisposing factors for infection in spine surgery: a survey of 850 spinal procedures. J Spinal Disord. 1998;11(2):124-8.

31.Wimmer C, Gluch H. Management of postoperative wound infection in posterior spinal fusion with instrumentation. J Spinal Disord. 1996;9(6):505-8.

32.Garrido E. Closed irrigation-suction technique in the treatment of lumbar laminectomy infection: case report. Neurosurgery. 1979;5(3):354-5.
33.Ido K, Shimizu K, Nakayama Y, Shikata J, Matsushita M, Nakamura T. Suction/irrigation for deep wound infection after spinal instrumentation: a case study. Eur Spine J. 1996;5(5):345-9.

34. Transfeldt E, Lonstein J, Winter R. Wound infections in reconstructive spinal surgery. Orthop Trans Rel Res. 1985;9:128-9.

35.Merrit K, Dowd JD. Role of internal fixation in infection of open fractures: studies with Staphylococcus aureus e Proteus mirabilis. J Orthop Res. 1987;5(1):23-8.

\section{Correspondência}

Asdrubal Falavigna

Rua General Arcy da Rocha Nóbrega, 401, sala 602

CEP 95040-290 - Caxias do Sul (RS), Brasil

Fone: 54 3222-0684

E-mail: asdrubal@doctor.com 